\title{
Human umbilical cord mesenchymal stem cells ameliorate acute liver failure by inhibiting apoptosis, inflammation and pyroptosis
}

\author{
Mengting Liu ${ }^{1,2}$, Jing $\mathrm{He}^{1}$, Shuo Zheng ${ }^{2}$, Ke Zhang ${ }^{1}$, Yu Ouyang ${ }^{1}$, Yaqi Zhang ${ }^{1}$, Changyong Li $^{3}$, \\ Dongcheng $\mathrm{Wu}^{1,2,4}$
}

${ }^{1}$ Department of Biochemistry and Molecular Biology, Wuhan University School of Basic Medical Sciences, Wuhan, China; ${ }^{2}$ R\&D Center, Wuhan Hamilton Biotechnology Co., Ltd, Wuhan, China; ${ }^{3}$ Department of Physiology, Wuhan University School of Basic Medical Sciences, Wuhan, China; ${ }^{4}$ R\&D Center, Guangzhou Hamilton Biotechnology Co., Ltd, Guangzhou, China

Contributions: (I) Conception and design: M Liu, C Li, D Wu; (II) Administration support: All authors; (III) Provision of study materials or patients: M Liu, J He, S Zheng; (IV) Collection and assembly of data: M Liu, K Zhang, Y Ouyang; (V) Data analysis and interpretation: M Liu, C Li; (VI) Manuscript writing: All authors; (VII) Final approval of manuscript: All authors.

Correspondence to: Changyong Li. Department of Physiology, Wuhan University School of Basic Medical Sciences, 185 Donghu Road, Wuhan 430071, China. Email: lichangyong@whu.edu.cn; Dongcheng Wu. Department of Biochemistry and Molecular Biology, Wuhan University School of Basic Medical Sciences, 185 Donghu Road, Wuhan 430071, China. Email: bcdcwu@hotmail.com.

Background: Human umbilical cord mesenchymal stem cells (UC-MSCs) are multipotent progenitor cells representing an attractive therapeutic tool for tissue damage and inflammation owing to their unique immunomodulatory properties. This study was designed to determine the protective effects and underlying mechanisms of UC-MSCs on acute liver failure (ALF).

Methods: ALF was induced in mice by intraperitoneal injection of D-galactosamine (D-GalN) and lipopolysaccharide (LPS). Mice were intravenously injected with $1 \times 10^{6}$ UC-MSCs one hour before or six hours after D-GalN/LPS injection. Liver function was valued by serum biochemical parameters and hematoxylin-eosin staining. Inflammatory cytokine and chemokine levels were measured by real-time PCR, and inflammatory cells infiltration was observed by immunofluorescence staining. Hepatocyte apoptosis and pyroptosis related proteins were detected by western blot. Murine macrophage Raw264.7 in the presentation of LPS was treated with the UC-MSCs condition medium (UC-MSCs-CM), and then the levels of inflammatory cytokines and NOD-like receptor family pyrin domain containing 3 (NLRP3) inflammasome in Raw264.7 were measured.

Results: UC-MSCs significantly reduced the mortality, decreased serum alanine aminotransferase and aspartate aminotransferase levels, and improved the pathological damage. Moreover, UC-MSCs inhibited inflammatory cytokine and chemokine levels, especially TNF- $\alpha$, interleukins-6 (IL-6), IL- $1 \beta$, monocyte chemoattractant protein (MCP-1), CC-chemokines ligand 2 (CCL2), C-X-C motif ligand 2 (CXCL2), and reduced macrophage, neutrophil and T lymphocyte infiltration into the liver tissue. UC-MSCs also attenuated hepatocyte apoptosis, as evidenced by decreased TUNEL positive cells, increased Bcl-xl/ Bax protein ratio and downregulated cleaved caspase 3 levels. NLRP3 inflammasome activation, IL-1 $\beta$ maturation and cleaved caspase1 were suppressed by UC-MSC administration. Furthermore, the UC-MSCsCM reduced the levels of inflammatory cytokines and the activation of NLRP3 inflammasome in Raw264.7.

Conclusions: Our results demonstrated that UC-MSCs exerted therapeutic effects on ALF by inhibiting apoptosis, inflammation, and pyroptosis.

Keywords: Umbilical cord mesenchymal stem cells (UC-MSCs); acute liver failure (ALF); apoptosis; inflammasome; pyroptosis

Submitted Jun 03, 2021. Accepted for publication Sep 05, 2021.

doi: $10.21037 / \mathrm{atm}-21-2885$

View this article at: https://dx.doi.org/10.21037/atm-21-2885 


\section{Introduction}

Acute liver failure (ALF) is an uncommon but severe disease characterized by massive hepatic necrosis and variable inflammation. It leads to hepatic encephalopathy, severe infection, coagulation disorder and kidney failure and poses a threat to the lives (1). Many etiologies contribute to ALF, but the major causes are hepatitis A, B, and E virus infections mostly coming from developing countries and drug hepatotoxicity from developed countries (2). Liver transplantation has been considered as the most effective therapy for patients with ALF. However, the high medical cost, shortage of donor liver, and long-term use of immunosuppressive agents severely limit its application.

D-galactosamine (D-GalN)/lipopolysaccharide (LPS) causes overwhelming inflammatory response, which can result in ALF (3). Pyroptosis is a lytic type of cell death that is initiated by inflammatory caspases. NOD-like receptor family pyrin domain containing 3 (NLRP3) inflammasome particles, containing NLRP3, apoptosis-associated specklike protein (ASC) and pro-caspase1 activates caspase1, mediates the release of matured interleukins- $1 \beta$ (IL-1 $\beta$ ) and IL-18, and plays a vital role in the inflammatory response (4). The activation of the inflammasome and IL- $1 \beta$ generate toxicity in acetaminophen-induced acute liver injury (5). Recent studies suggest that the protein gasdermin D (GSDMD) is a newly executioner of pyroptosis. Cleaved $\mathrm{N}$-terminal GSDMD (GSDMD-N), as an active form was identified to trigger pyroptosis in several diseases, such as sepsis (6), non-alcoholic steatohepatitis (7) and acute kidney injury (8).

Mesenchymal stem cells (MSCs) have self-renewing ability and multiple differentiation potentials to various types, including hepatocytes (9). More importantly, MSCs possess immunosuppressive properties and the ability to secrete various trophic factors $(10,11)$. MSCs can be isolated from multiple human body sites, such as bone marrow, adipose, placental tissue, and umbilical cord (12). Human umbilical cord MSCs (UC-MSCs) present much superiority compared to stem cells from other sources, including non-invasive harvest procedure, rapid proliferation, low immunogenicity, and no appreciable ethical issues (13). Currently, MSCs have been identified as a promising therapy for liver diseases $(14,15)$. Studies demonstrated that MSCs attenuated acute or chronic liver injuries $(16,17)$. UC-MSCs significantly improved liver function in patients with liver cirrhosis (18). However, the underlying mechanism of the encouraging effects has not been completely clarified.

In the present study, we systematically evaluated the therapeutic effects of UC-MSCs on D-GalN/LPSinduced ALF in a murine model. Our data revealed UCMSC administration attenuated ALF through inhibiting apoptosis, inflammation and pyroptosis. We present the following article in accordance with the ARRIVE reporting checklist (available at https://dx.doi.org/10.21037/atm-212885).

\section{Methods}

\section{Preparation of UC-MSCs and UC-MSCs condition medium}

The study was conducted in accordance with the Declaration of Helsinki (as revised in 2013). The use of the human umbilical cord tissue from a healthy donor who gave birth and signed informed consent in Renmin Hospital was supported by the Institutional Ethics Review Board of Renmin Hospital of Wuhan University (Permit Number: WDRY2019-G001). UC-MSCs were isolated, cultured and identified as described in our previous study (19). Briefly, the umbilical cord was cut into small pieces and cultured in serum-free medium (UltraCULTURE, Walkersville Lonza, MD) supplemented with serum alternatives (Life Science, France) and L-Glutamine at $37^{\circ} \mathrm{C}$ in a humidified atmosphere containing $5 \% \mathrm{CO}_{2}$. The fifth generations of cells were used in this animal experiment. UC-MSCs were characterized for surface marker expression and differentiation potential of adipogenesis, osteogenesis, and chondrogenesis as described previously (19). For the production of UC-MSCs condition medium (UC-MSCsCM), the supernatant of UC-MSCs was collected and concentrated 25-fold using ultrafilter (Millipore, Bedford, MA), when the confluence reached about $80 \%$.

\section{Animals}

Male mice ( $\mathrm{n}=44$; C57BL/6J, 20-22 g) were purchased from Hubei Provincial Center for Disease Control and Prevention (Wuhan, China). All animals were housed in sterile cages with free access to food and water at $12 \mathrm{~h}$ light/ dark cycles. Animal welfare and experimental procedures were performed according to the guide for the Care and Use of Laboratory animals. A protocol was prepared before the study without registration. This study was approved by the Committee of Animals Care and Use of Hubei 
Provincial Center for Food and Drug Safety Evaluation and Animal Experiment (Permit Number: 2020020).

Mice were randomized into the following four groups: control group, UC-MSCs alone, D-GalN/LPS group, and D-GalN/LPS + UC-MSCs group (n=6/group). ALF was induced by intraperitoneal injection of D-GalN $(400 \mathrm{mg} / \mathrm{kg}$, Carl Roth, Karlsruhe, Germany) and LPS (10 $\mu \mathrm{g} / \mathrm{kg}$, SigmaAldrich, Hamburg, Germany). UC-MSCs $\left(1 \times 10^{6}\right)$ were administered via tail veil one hour prior to D-GalN/LPS injection. Blood and liver tissues were harvested at $6 \mathrm{~h}$ after D-GalN/LPS injection for evaluation of liver injury. We also assessed the effects of UC-MSCs on survival rate, initially at $6 \mathrm{~h}$ after D-GalN/LPS injection and then every hour over $24 \mathrm{~h}(\mathrm{n}=9$ in D-GalN/LPS group and $\mathrm{n}=10$ in D-GalN/LPS + UC-MSCs group).

To assess the therapeutic potential of UC-MSCs, administration of UC-MSCs at $6 \mathrm{~h}$ following ALF (D-GalN $200 \mathrm{mg} / \mathrm{kg}$, LPS $10 \mu \mathrm{g} / \mathrm{kg}$ ) were also examined. The mice were sacrificed $4 \mathrm{~h}$ after the UC-MSC administration.

\section{Serological analysis}

Alanine amino transferase (ALT) and aspartate amino transferase (AST) were measured to assess liver function using automated biochemistry analyzer (Chemray, Shenzhen, China) according to automated procedure.

\section{Quantitative real-time PCR}

Total RNA was extracted from cells or liver tissues with Trizol reagent (Life Technologies, USA), and $1 \mu \mathrm{g}$ of RNA was reverse transcribed into complementary first-strand cDNA using cDNA synthesis kit (Takara Bio, Inc., Otsu, Japan). The SYBR Green Mix (Applied Biosystems) was used for quantitative real-time PCR (qRT-PCR). Target gene expressions were calculated by their ratios to the housekeeping gene GAPDH. The sequences of primers were as follows:

TNF- $\alpha$ forward: 5 '-CTTCTGTCTACTGA ACTTCGGG-3', TNF- $\alpha$ reverse: 5'-TGATCTGAGTGT GAGGGTCTG-3';

IL-6 forward: 5' -TGATGGATGCTACCAAA CTGGA-3', IL-6 reverse: 5'-TGTGACTCCAGCTTAT CTCTTGG-3'; IL- $1 \beta$ forward: 5'-ACGGACCCCAAAA GATGAAG-3', IL- $1 \beta$ reverse: 5'-CACGGGAAAGAC ACAGGTAG-3'; Monocyte chemoattractant protein-1 (MCP-1) forward: 5'-CTCACCTGCTGCTAC TCATTC-3', MCP-1 reverse: 5'-TTACGGCTCAACTT
CACATTCA-3'; CC-chemokines ligand 2 (CCL2) forward: 5'-CTTCTGGGCCTGCTGTTCA-3', CCL2 reverse: 5'-CCAGCCTACTCATTGGGATCA-3'; C-X-C motif ligand 2 (CXCL2) forward: 5' CTCTCAAGGGCGGTCAAAAAGTT-3', CXCL2 reverse: 5'-TCAGACAGCGAGGCACATCAGGTA-3'; GAPDH forward: 5'-AGTATGTCGTGGAGTCTACTG GTGT-3', GAPDH reverse: 5'-AGTGAGTTGTCATAT TTCTCGTGGT-3'.

\section{Cytokine detection by ELISA}

The levels of IL-1 $\beta$, TNF- $\alpha$ and IL-18 in the serum and/ or liver tissue were measured by ELISA according to the manufacturer's instructions (Mlbio, Shanghai, China).

\section{Histopathology, immunobistochemistry and immunofluorescence}

Fresh liver tissues were fixed with $10 \%$ formalin, embedded in paraffin and sectioned into $4 \mu \mathrm{m}$ sections. Sections were deparaffinized in xylene, rehydrated in alcohol and distilled water, and then routinely stained with hematoxylin and eosin (H\&E staining).

After deparaffinized and rehydrated, liver sections were processed for antigen retrieval in a pressure cooker with EDTA buffer (pH 8.0) for immunofluorescence staining. The slides were washed in PBS for 3 times. Fluorescent quencher was added to avoid spontaneous fluorescence and then rinsed off in running buffer. BSA was dropped onto the section and incubated for $30 \mathrm{~min}$. Specific primary antibodies against F4/80 (1:1,000; Cell Signaling Technology, USA), CD3 (1:2,000; Abcam, USA), Ly6G (1:200; Bioss, China) and CD11b (1:3,000; Abcam, USA) were added and incubated overnight at $4{ }^{\circ} \mathrm{C}$. After washing with PBS for 3 times, the sections were incubated with CY3-conjugated anti-rabbit or anti-mouse IgG (1:400; Thermo, USA), and FITC-conjugated anti-mouse IgG (1:400; Abcam, USA). The nucleus was stained with 4', 6-diamidino-2-phenylindole (DAPI; Solarbio, Beijing, China)

\section{TUNEL assay}

TUNEL assay was carried out according to the manufacturer's instructions. Briefly, deparaffinized, rehydrated and retrieved sections were treated with $0.1 \%$ Triton X-100 for $20 \mathrm{~min}$ at room temperature, blocked 
with $3 \% \mathrm{H}_{2} \mathrm{O}_{2}$, washed in PBS for 3 times and then placed in working solution (10\% enzyme solution and $90 \%$ label solution) for $2 \mathrm{~h}$ at $37{ }^{\circ} \mathrm{C}$. Diaminobenzidine (Solarbio, Beijing, China) substrate was added, and the apoptotic cells were observed under a microscope.

\section{Western blot analysis}

Snapped frozen liver tissues were treated with RIPA lysis buffer for protein extraction (Beyotime, Nanjing, China). Protein concentrations were determined using the BCA assay (Beyotime, Nanjing, China). Proteins $(60 \mu \mathrm{g})$ were loaded into sodium dodecyl sulfate-polyacrylamide gel electrophoresis (SDS-PAGE) for separation and then transferred onto PVDF membranes (Millipore, Bedford, MA, USA). The membranes were blocked with $5 \%$ nonfat milk at room temperature and incubated overnight at $4{ }^{\circ} \mathrm{C}$ with primary antibodies against NLRP3 (1:1,000; Cell Signaling Technology, USA), ASC (1:1,000; Santa Cruz, USA), Cleaved caspase1 (1:1,000; Santa Cruz, USA), Bcl-xl (1:1,000; Cell Signaling Technology, USA), Bax (1:1,000; Cell Signaling Technology, USA), Cleavedcaspase3 (1:1,000; Cell Signaling Technology, USA) and GAPDH (1:2,000; Proteintech, China). After 3 washes, the membranes were incubated with horseradish peroxidase (HRP)-conjugated anti-rabbit or anti-mouse IgG (1:10,000; Proteintech, China) at room temperature for $1 \mathrm{~h}$. The proteins bands were developed using Electrochemiluminescence (Biorad, USA) reagents and imaged using the Imaging System (Peiqing, Shanghai, China). The gray value was quantified using Image $\mathrm{J}$ software.

\section{Co-culture of macrophages and UC-MSCs}

Raw264.7 cells were suspended in DMEM medium with $10 \%$ fetal bovine serum and plated in a 6 -well plate at a density of $1 \times 10^{5}$ cell per well. LPS $(100 \mathrm{ng} / \mathrm{mL})$ was used to activate the Raw264.7 in DMEM medium. Raw264.7 cells under the LPS stimulation were treated by adding $80 \%$ UC-MSCs-CM. After $24 \mathrm{~h}$, cells were collected for RNA and protein extraction and analysis.

\section{Statistical analysis}

All data are presented as mean $\pm \mathrm{SD}$ and analyzed by two- tailed Student's $t$-test. Multiple group comparisons were made using one-way ANOVA followed by Bonferroni's post hoc test (SPSS Inc., Chicago, Illinois). Survival data were analyzed by Kaplan-Meier curves with $\mathrm{P}$ values assessed with log-rank (Mantel-Cox) test using GraphPad Prism (GraphPad Software, Inc, La Jolla, USA). P value $<0.05$ was considered as statistically significant.

\section{Results}

\section{UC-MSC administration reduced liver injury and mortality in D-GalN/LPS-induced ALF}

A mouse model of ALF was induced by intraperitoneal injection of D-GalN/LPS. UC-MSCs with $98 \%$ cell viability were administered through the tail vein one hour before GalN/LPS injection. Mice were sacrificed, and liver tissue and blood were collected $6 \mathrm{~h}$ after D-GalN/LPS injection (Figure 1A). To determine the efficacy of UCMSCs in ALF mice, we recorded the survival rate. The survival rate of UC-MSCs-treated mice was significantly greater than that of the D-GalN/LPS group (Figure 1B). We also observed gross morphology of the liver. Liver hyperemia and enlargement were the obvious features in the D-GalN/LPS group, and the injury extent was prevented in the UC-MSC treatment group (Figure 1C). Meantime, the liver/body weight ratio was significantly decreased with UC-MSC treatment compared to the D-GalN/LPS group (Figure 1D).

The hepatocellular function as measured by ALT and AST levels was significantly improved after UCMSC administration in mice compared with untreated controls (Figure 1E). Consistent with these functional data, liver histology showed that UC-MSC treatment in mice significantly attenuated D-GalN/LPS-induced liver injury (Figure $1 F$ ). Notably, there was no difference between the control group and the UC-MSCs alone, which suggested the safety of UC-MSC injection in mice. These results indicated that UC-MSCs ameliorated hepatic injury and increased the survival rate of ALF mice induced by D-GalN/LPS.

To evaluate the therapeutic effects of UC-MSCs, we intravenously gave UC-MSCs $6 \mathrm{~h}$ after D-GalN/LPS injection. The blood and liver were collected $4 \mathrm{~h}$ after the UC-MSC therapy. H\&E staining showed that liver injury were lessoned, and the levels of ALT and AST were significantly reduced in mice with UC-MSC therapy (Figure S1A,S1B). 
A

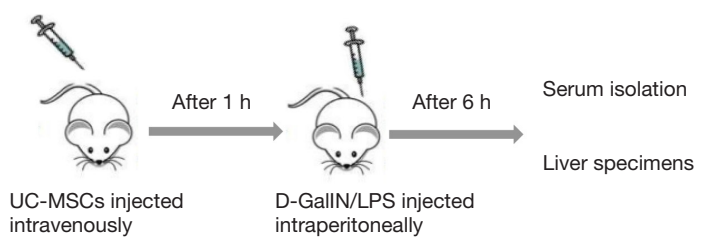

C

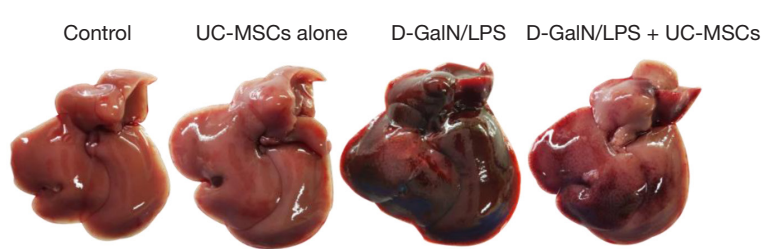

$\mathrm{F}$
UC-MSCs alone

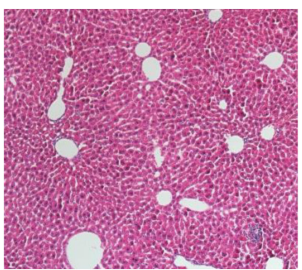

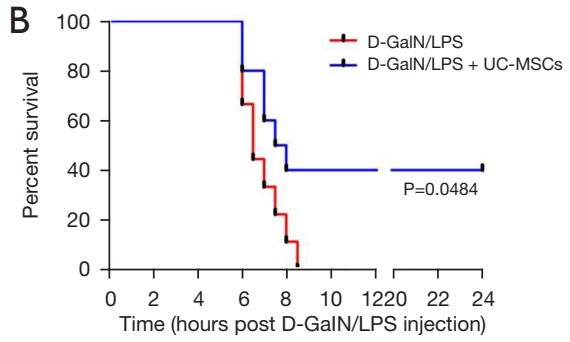

D

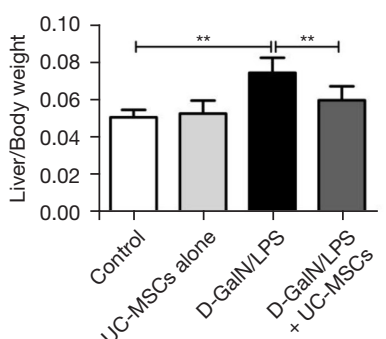

E

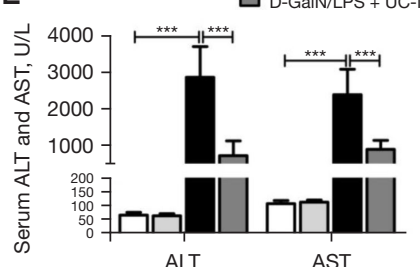

D-GaIN/LPS + UC-MSCs
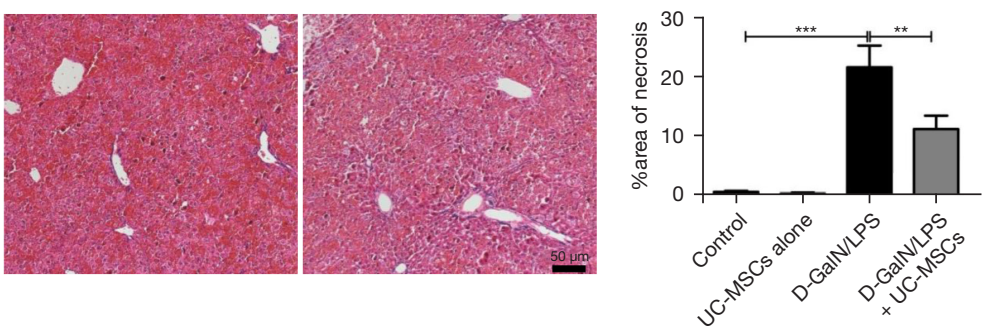

Figure 1 UC-MSCs attenuate liver injury in D-GalN/LPS-induced ALF mice. (A) Schematic representation of the study design. Mice were treated with D-GalN/LPS (400 mg/kg and $10 \mu \mathrm{g} / \mathrm{kg}$ respectively) or PBS and liver injury was assessed after $6 \mathrm{~h}$. UC-MSCs $\left(1 \times 10^{6}\right)$ were administered via tail veil one hour prior to D-GalN/LPS injection ( $\mathrm{n}=6$ mice/group). (B) The survival rate of mice presented by KaplanMeier curve (n=9-10 mice/group). (C) The gross appearance of the collected liver tissues from control, UC-MSCs alone, and D-GalN/LPS mice with or without US-MSC administration. (D) The liver/body weight ratio. (E) The levels of serum ALT and AST. (F) Hematoxylineosin staining of liver tissues from the control group, UC-MSCs alone, D-GalN/LPS group and D-GalN/LPS + UC-MSCs group. Scale bar: $50 \mu \mathrm{m} .{ }^{* *}, \mathrm{P}<0.01$; ***, $\mathrm{P}<0.001$. D-GalN, D-galactosamine; LPS, lipopolysaccharide; ALF, acute liver injury; UC-MSCs, umbilical cord mesenchymal stem cells; ALT, alanine amino transferase; AST, aspartate amino transferase.

\section{UC-MSC administration decreased D-GalN/LPS-induced bepatocyte apoptosis}

Massive hepatocyte apoptosis was another important pathogenic component leading to ALF. TUNEL assay confirmed extensive hepatocyte apoptosis in the D-GalN/ LPS group, while apoptotic hepatocytes were considerably reduced by UC-MSC administration $1 \mathrm{~h}$ before or $6 \mathrm{~h}$ after D-GalN/LPS injection (Figure $2 A$ and Figure S1C). The level of TNF- $\alpha$, a critical apoptotic inducing cytokine, was significantly decreased in serum from UC-MSCs-treated mice (Figure 2B). The ratio of anti-apoptotic protein Bcl-xl and pro-apoptotic protein Bax was markedly decreased in the
D-GalN/LPS group compared with the control group, while the ratio was increased in the mice with UC-MSC treatment. Moreover, UC-MSC treatment in mice significantly reduced a pro-apoptotic molecule, cleaved caspase 3 expression compared with the D-GalN/LPS group (Figure 2C). These data collectively indicated that the protective effects of UCMSCs against D-GalN/LPS-induced ALF could be related to reducing hepatocyte apoptosis.

\section{UC-MSC administration inhibited D-GalN/LPS-induced liver inflammation}

Inflammatory cytokine production and inflammatory 

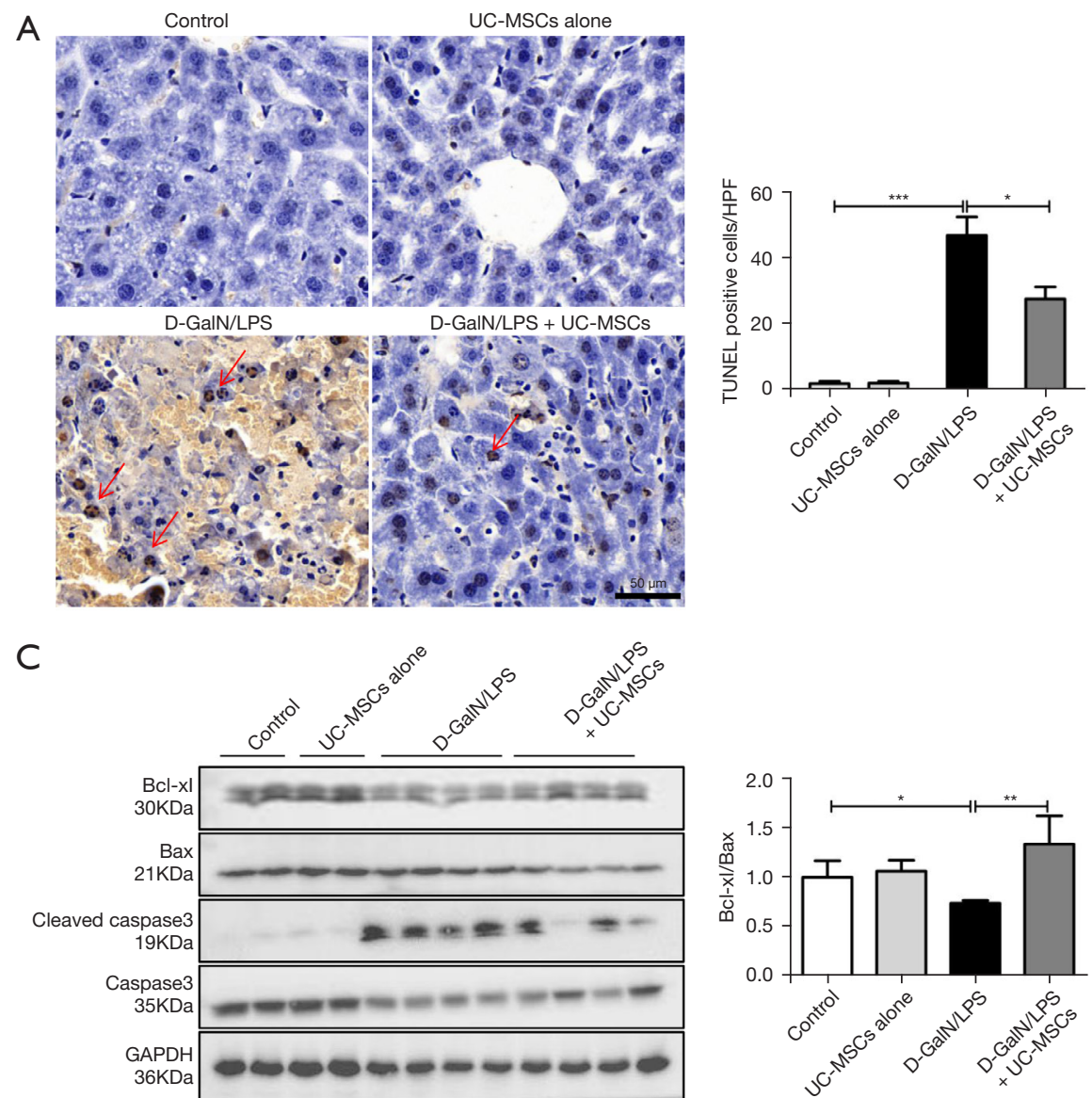

B
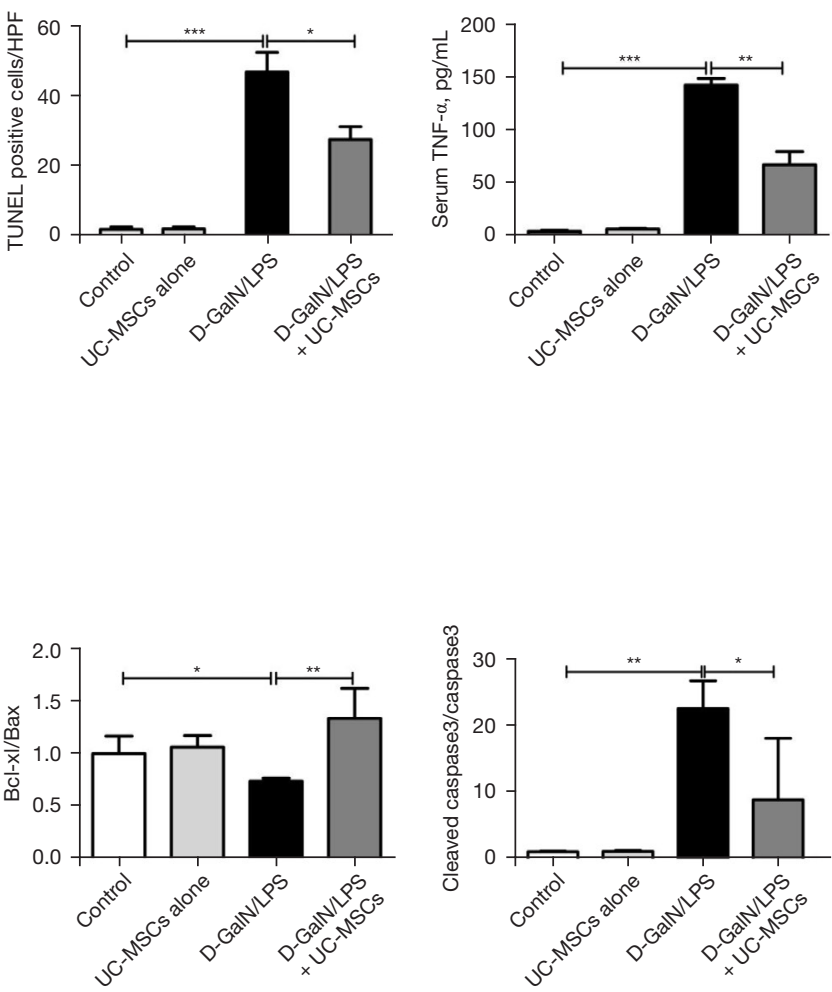

Figure 2 UC-MSCs alleviate hepatocyte apoptosis in liver tissue. (A) Hepatocyte apoptosis was detected by immunohistochemistry staining. Scale bar: $50 \mu \mathrm{m}$. The apoptotic cells were pointed the by red arrow and counted in randomly 5 chosen histological fields per slide ( $\mathrm{n}=6$ mice/group). (B) ELISA analysis of TNF- $\alpha$ levels in animal serum ( $\mathrm{n}=6$ mice/group). (C) Protein levels of Bcl-xl, Bax, Cleaved caspase 3 and Caspase 3 in the liver tissue were measured by Western blot and quantified by gray value assay. ${ }^{*}, \mathrm{P}<0.05 ;{ }^{* *}, \mathrm{P}<0.01 ;{ }^{* * *}, \mathrm{P}<0.001$. $\mathrm{UC}-$ MSCs, umbilical cord mesenchymal stem cells; ELISA, enzyme-linked immunosorbent assay; Bcl-xl, B-Cell Lymphoma-extra-large.

cell infiltration were reported to play a vital role in the pathogenesis of ALF. As expected, mRNA levels of TNF- $\alpha$, IL-6, IL-1 $\beta$, MCP-1, CCL2, and CXCL2 in liver tissue were significantly decreased in the UC-MSC treatment group compared with the D-GalN/LPS group (Figure 3A). Immunofluorescence revealed that $\mathrm{F} 4 / 80^{+}$macrophages, Ly6G ${ }^{+}$neutrophils, $\mathrm{CD}^{+} \mathrm{T}$ lymphocytes and $\mathrm{CD} 11 \mathrm{~b}^{+}$ macrophages were largely gathered in the liver from D-GalN/LPS-treated mice, while UC-MSC administration markedly reduced these inflammatory cell infiltrations (Figure $3 B$ ). CD $11 \mathrm{~b}^{+}$monocyte-derived macrophage were also reduced by UC-MSC administration $6 \mathrm{~h}$ after D-GalN/ LPS injection (Figure S1D). These results demonstrated that UC-MSCs could reduce liver inflammation during D-GalN/LPS-induced ALF.

\section{UC-MSC administration inbibited NLRP3 inflammasome activation}

The activation of the NLRP3 inflammasome was supposed to be involved in the development of ALF (5). Therefore, we investigated the effect of UC-MSCs on NLRP3 inflammasome activation in ALF mice. The expression of NLRP3, Cleaved caspase1, ASC and GSDMD-N proteins were significantly decreased in the UC-MSC treatment group compared with those in the D-GalN/LPS group (Figure $4 A$ ). The levels of IL-1 $\beta$ and IL-18 in liver tissue (Figure $4 B, 4 C$ ) were markedly increased in the D-GalN/ LPS-induced mice and significantly lowered in UC-MSC treatment mice. These results suggested that UC-MSCs reduced NLRP3 inflammasome activation and subsequent 
A
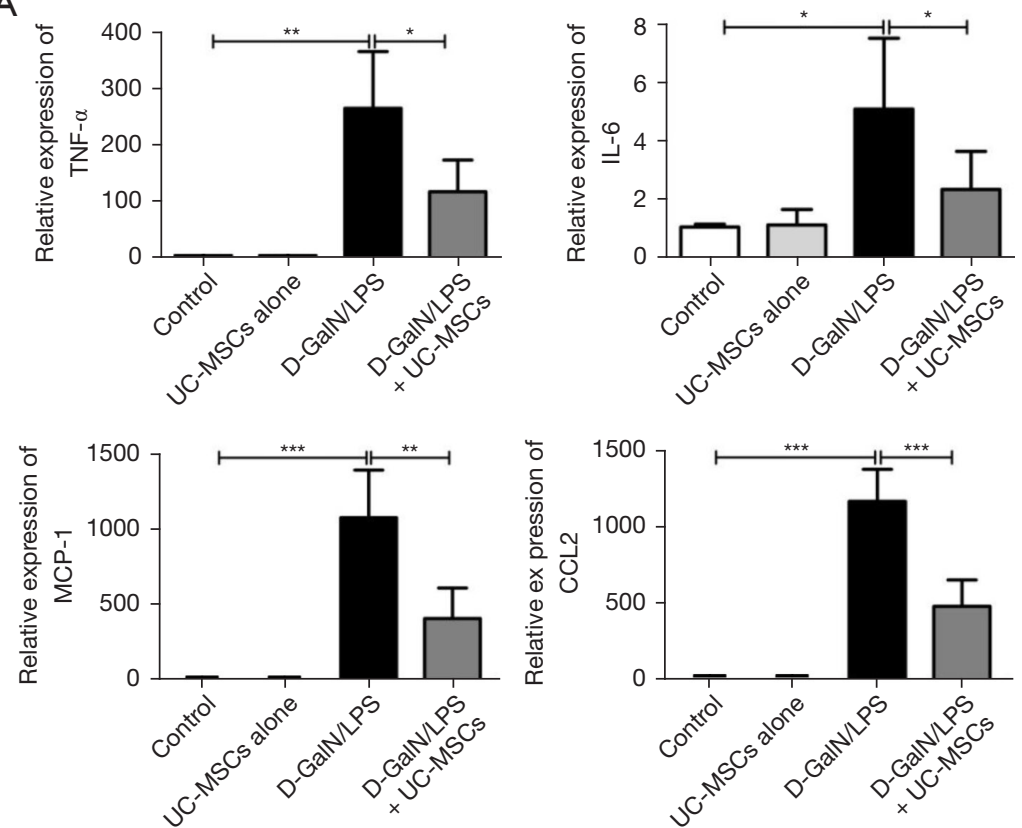

B

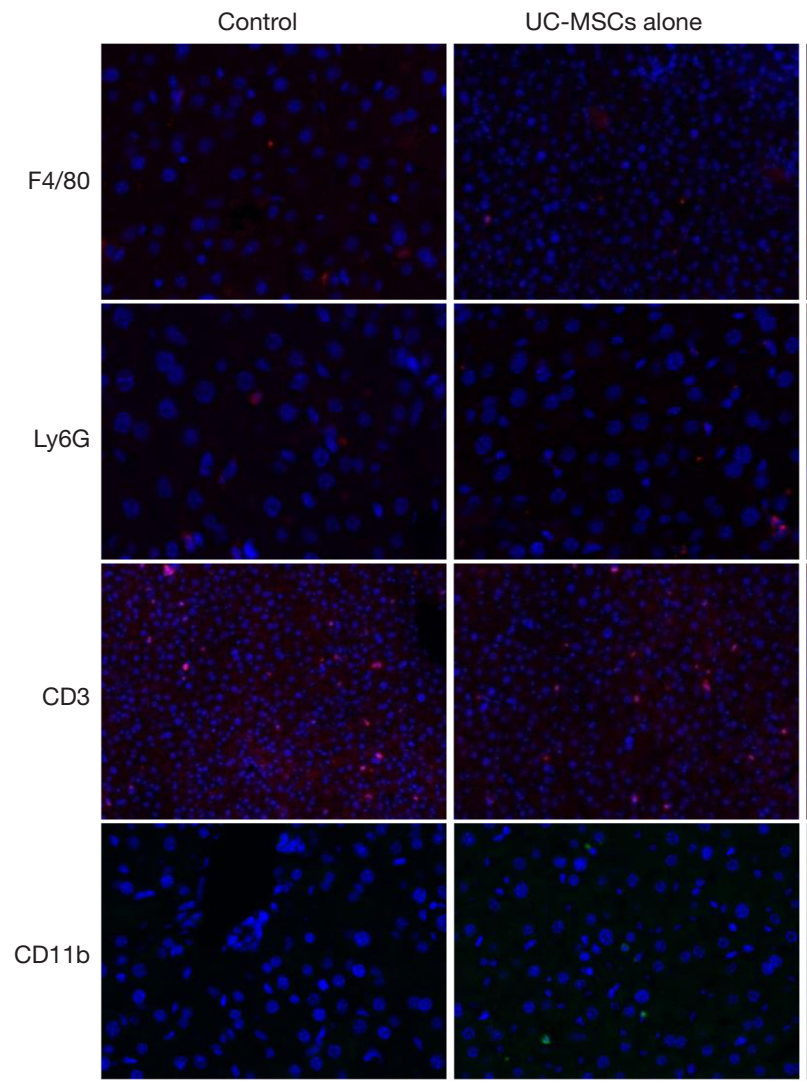

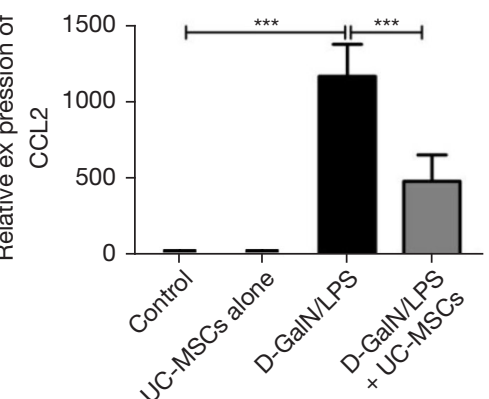

D-GaIN/LPS
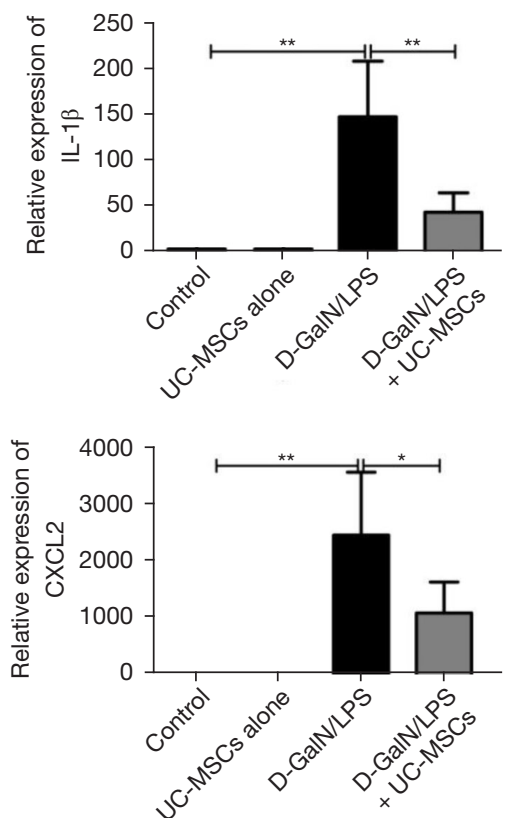

D-GalN/LPS + UC-MSCs

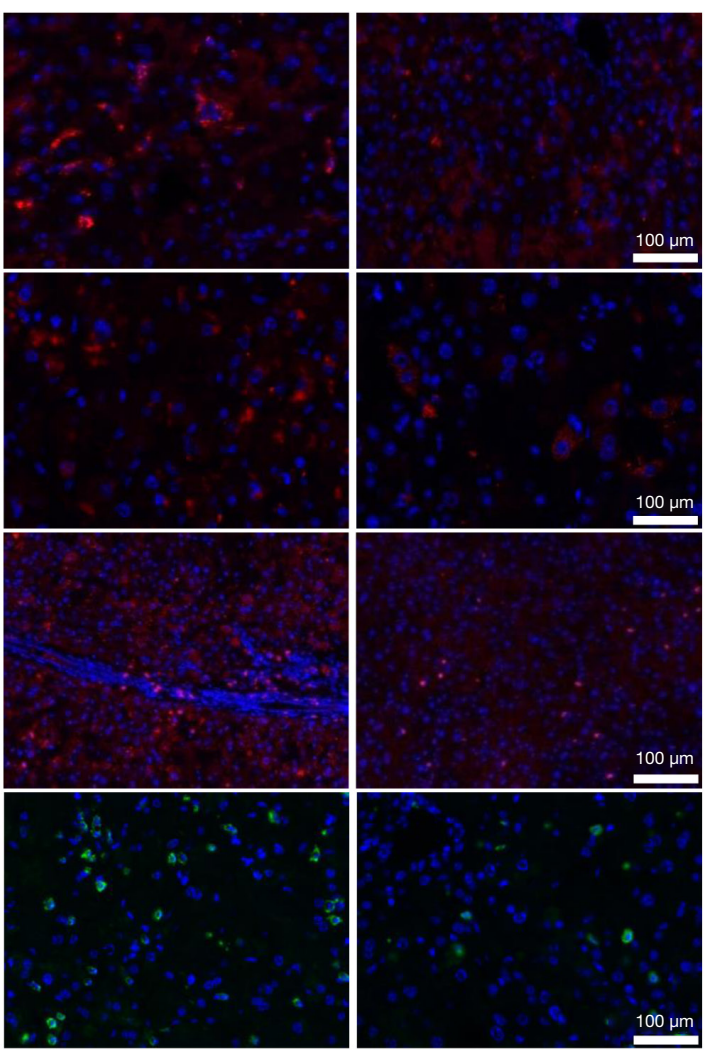



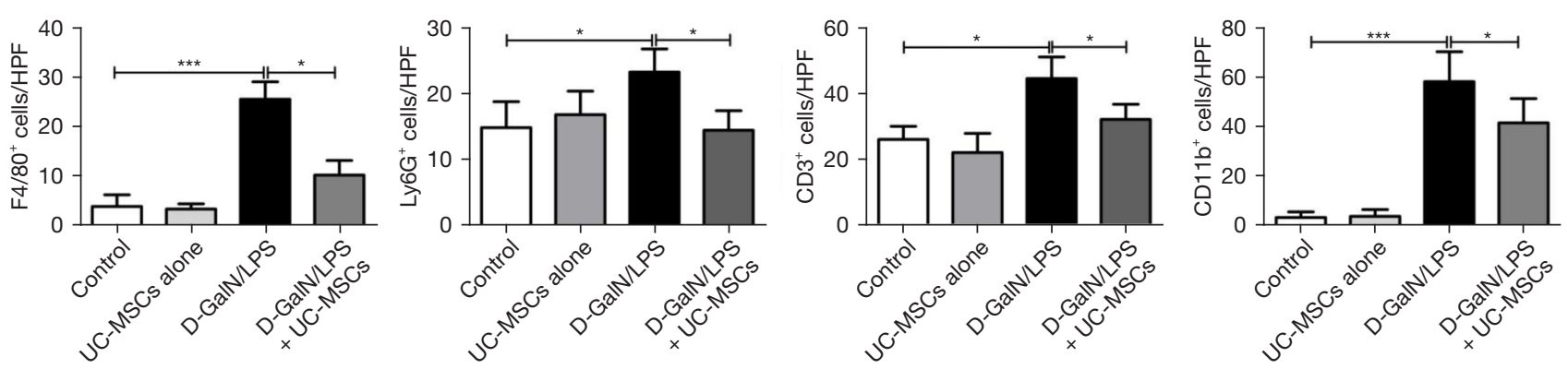

Figure 3 UC-MSCs reduce inflammatory cytokine and chemokine levels and inflammatory cell infiltration. (A) Hepatic mRNA levels of inflammatory cytokines TNF- $\alpha$, IL-6, IL-1 $\beta$ and chemokines MCP-1, CCL2 and CXCL2 were detected by RT-qPCR. (B) Representative immunofluorescence staining of the macrophage marker F4/80 $0^{+}$and $\mathrm{CD}_{11 \mathrm{~b}}{ }^{+}$, neutrophil marker Ly6 $\mathrm{G}^{+}$, and $\mathrm{T}$ lymphocyte marker CD3 + in liver sections. CY3-conjugated (red) or FITC-conjugated (green) secondary antibodies were used. DAPI was used to visualize nuclei (blue). Quantification of $\mathrm{F} 4 / 80^{+}, \mathrm{Ly}_{6 \mathrm{G}}{ }^{+}, \mathrm{CD}^{+}$and $\mathrm{CD} 11 \mathrm{~b}^{+}$per high power field. Scale bar: $100 \mu \mathrm{m} .{ }^{*}, \mathrm{P}<0.05{ }^{* *}, \mathrm{P}<0.01$ and ${ }^{* * *}$, $\mathrm{P}<0.001$. UC-MSCs, umbilical cord mesenchymal stem cells; MCP-1, monocyte chemoattractant protein; CCL2, CC-chemokines ligand 2; CXCL2, C-X-C motif ligand 2; RT-qPCR, real-time quantitative polymerase chain reaction; FITC, fluorescein isothiocyanate; DAPI, 4',6-diamidino-2-phenylindole.

pyroptotic cell death.

\section{UC-MSCs reduced inflammatory response in macrophages in vitro}

To further evaluate the anti-inflammatory effects of UCMSCs, in vitro experiments were performed in a mouse macrophage cell line Raw264.7 cells. UC-MSCs-CM was collected to co-culture with LPS-primed Raw264.7. As shown in Figure $5 \mathrm{~A}$, inflammatory cytokines including TNF- $\alpha$, IL-6, IL-1 $\beta$, MCP-1, CCL2, and CXCL2 were reduced by the UC-MSCs-CM treatment. NLRP3 and GSDMD-N expression were also decreased in Raw264.7 cultured with UC-MSCs-CM compared with untreated controls (Figure 5B). These results suggested that UCMSCs suppressed inflammatory response in macrophage via a paracrine mechanism.

\section{Discussion}

ALF is characterized by a sudden and severe impairment of liver function. Massive hepatocellular necrosis and inflammation storm are distinguishing features leading to the final liver failure. Due to the diversity of the pathogenesis and high mortality, the clinical management of ALF is particularly difficult. In the current study, we demonstrated that UC-MSCs suppressed the local and systemic inflammatory cytokine and chemokine levels, reduced inflammatory cell infiltration and hepatocyte apoptosis, inhibited NLRP3 inflammasome activation, and decreased mortality from ALF induced by D-GalN/LPS.

The liver receives the majority of its blood from the gut and represents an essential line of defense against products from the digestive tract, underlining the implication of gut-derived endotoxins in the pathogenesis of acute liver injury. D-GalN/LPS-induced ALF is a well-established model to evaluate the efficiency of therapeutic drugs, including the stem cells drug. Transplantation of MSCs improved liver function and prolonged the survival time of swine with ALF (20). Human amniotic MSCs improved acetaminophen-induced acute liver injury (21). Furthermore, exogenous MSCs were proved to survive in a mice body and homed to the injured liver to exert hepatoprotective function (22). In this study, we explored the time point of UC-MSC administration: $1 \mathrm{~h}$ before and $6 \mathrm{~h}$ after D-GalN/LPS injection. We found the two intervention timing had therapeutic effects in D-GalN/ LPS-injected mice. These findings suggested that UCMSCs might have a potential clinical application in ALF. It should be noted that the effects of UC-MSCs on ALF induced by viruses or drug needed to be explored.

Hepatocyte apoptosis was reported to be a consequence of over-activated immune response and often mediated by TNF- $\alpha$ (23). TNF- $\alpha$ interacted with its receptor TNF receptor $\mathrm{I}$ and then activated the signal transduction pathway (24). Caspase 3 was activated through apoptosis 

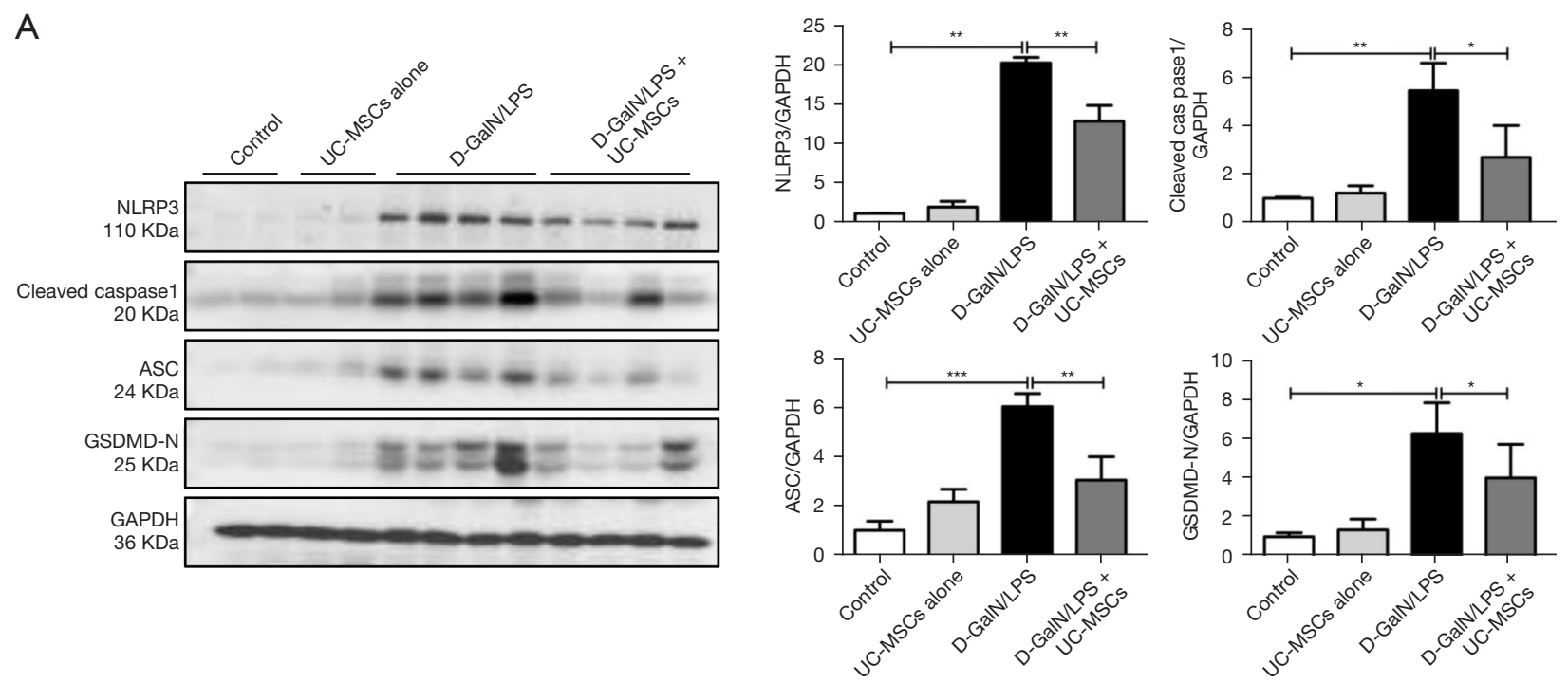

B

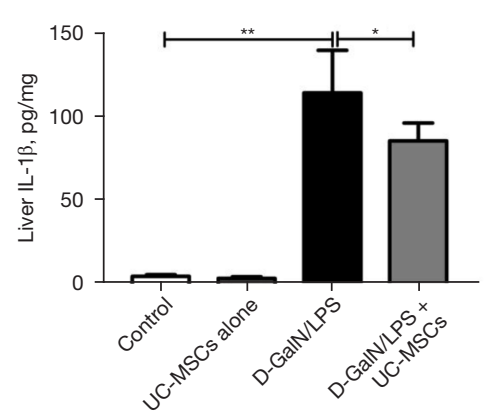

C

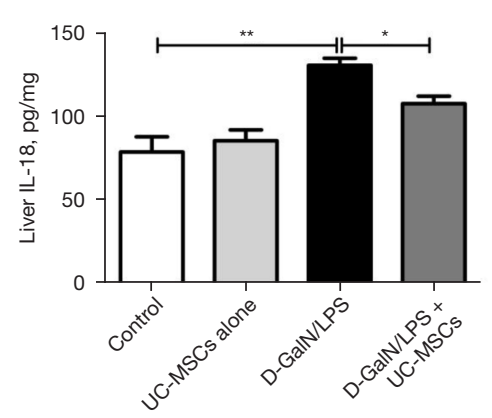

Figure 4 UC-MSCs suppress NLRP3 inflammasome activation. (A) Effects of UC-MSCs on the expression levels of inflammasome associated proteins in liver samples by Western blot analysis. NLRP3, cleaved caspase1, ASC and GSDMD-N expressions were quantified by gray value assay. The concentration of IL- $1 \beta(\mathrm{B})$ and IL-18 (C) in liver tissue was measured by ELISA. *, $\mathrm{P}<0.05$; **, $\mathrm{P}<0.01$ and ${ }^{* * *}$, $\mathrm{P}<0.001$. UC-MSCs, umbilical cord mesenchymal stem cells; ELISA, enzyme-linked immunosorbent assay; NLRP3, NOD-like receptor family pyrin domain containing 3; ASC, apoptosis-associated speck-like protein; GSDMD-N, gasdermin D N-terminal.

signal pathway (25). The pro- and anti-apoptotic Bcl-2 family proteins, including Bax, Bcl-2 and Bcl-xl regulated apoptosis depended on the mitochondrial pathway (26). Clearly, the level of TNF- $\alpha$ in serum, apoptotic hepatocytes and activated caspase 3 were significantly increased after D-GalN/LPS injection. Our results suggested that TNF- $\alpha$ production was involved in hepatocyte apoptosis in D-GalN/LPS-induced ALF.

It is well known that the liver is highly enriched with innate immune cells and plays a key role in the host defense and the regulation of inflammation. Previous study showed that whatever the etiologies were, uncontrolled inflammation was crucial to the initiation and progression of ALF (27). In the plasma of ALF patients, TNF- $\alpha$,
IL-6 and IL-1 $\beta$ levels were significantly increased (28). Release of inflammatory cytokines facilitated macrophage/ neutrophil infiltration during the pathogenesis of ALF (29). We found that D-GalN/LPS injection resulted in a surge of inflammatory cytokines and chemokines, as well as a rise in inflammatory cell recruitment. UC-MSCs significantly inhibited the expression of these inflammatory cytokines and chemokines. UC-MSCs also alleviated F4/80 macrophage, Ly6G ${ }^{+}$neutrophil and $\mathrm{CD}^{+} \mathrm{T}$ lymphocyte infiltration. Notably, studies highlighted macrophages were especially relevant to the pathogenesis of acute liver injury (30). In this line, our current study determined the effects of UC-MSCs on macrophages in vitro. We found that UC-MSCs-CM significantly inhibited pro- 

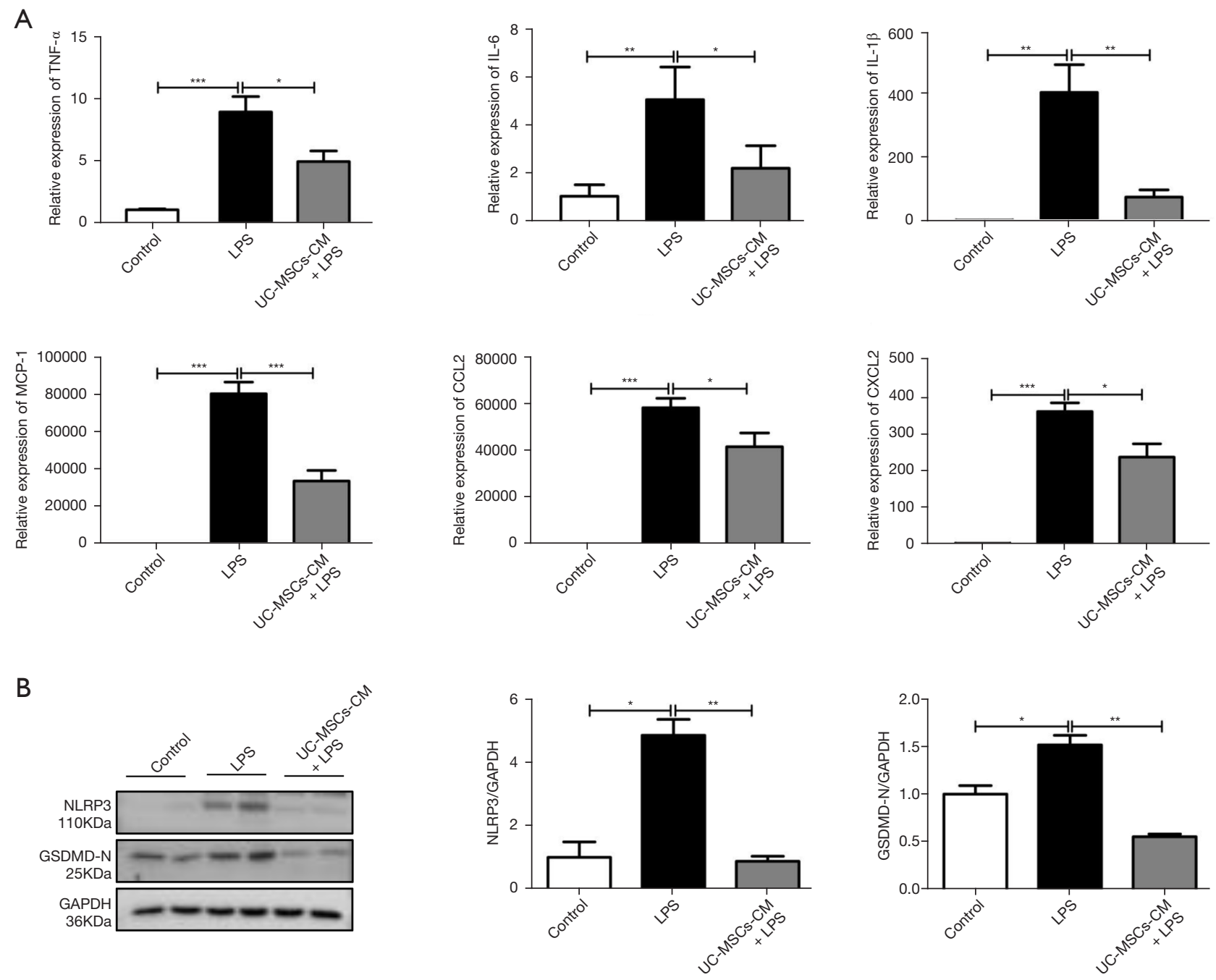

Figure 5 UC-MSCs-CM reduces the inflammatory response of Raw264.7 cells. UC-MSCs-CM transferred to the Raw264.7 cultures, and cultured for $24 \mathrm{~h}$ in the presence of LPS stimulation. (A) mRNA levels of inflammatory cytokines TNF- $\alpha$, IL-6, IL-1 $\beta$ and chemokines MCP-1, CCL2 and CXCL2 were detected by RT-qPCR. (B) Western blot analysis of protein NLRP3 and GSDMD-N in Raw264.7. *, $\mathrm{P}<0.05$; ** $\mathrm{P}<0.01$ and ***, $\mathrm{P}<0.001$. UC-MSCs, umbilical cord mesenchymal stem cells; MCP-1, monocyte chemoattractant protein; CCL2, CC-chemokines ligand 2; CXCL2, C-X-C motif ligand 2; NLRP3, NOD-like receptor family pyrin domain containing 3; GSDMD-N, gasdermin D-N-terminal; RT-qPCR, real-time quantitative polymerase chain reaction.

inflammatory cytokine and chemokine levels in Raw 264.7 cells with LPS stimulation. It's well accepted that numerous soluble factors in UC-MSCs-CM were associated with the improvement of liver injury (31). The exosomes from MSCs-CM also played a therapeutic role in various liver diseases (32). Therefore, we speculated that the therapeutic effects may be the combined effects. The exact mechanisms needed further investigation.

Inflammasome activation has been identified as a critical contributor to hepatocyte damage and immune cell amplification. IL- $1 \alpha / \beta$ and ROS production trigger NLRP3, ASC and pro-caspase 1 assembly into a complex (25). The complex leads to the maturation and secretion of inflammatory cytokines IL-1 $\beta$ and IL-18 (33). Importantly, pyroptosis is accompanied by inflammasome-induced inflammatory caspases in the caspase- 1 family (34). Here, we observed that UC-MSCs significantly suppressed D-GalN/LPS-induced inflammasome activation, which was 
determined by the expressions of NLRP3, cleaved caspase1, ASC and IL-1 $\beta$ secretion in the liver and serum. Moreover, UC-MSC treatment reduced the expression of GSDMD-N, an effector of pyroptosis. These results suggested that the inhibition of inflammasome activation contributed to the therapeutic effects of UC-MSCs on ALF.

\section{Conclusions}

The present study demonstrated that UC-MSCs could ameliorate D-GalN/LPS-induced ALF through inhibiting apoptosis, inflammation, and pyroptosis, indicating the significant potential of UC-MSCs used as an attractive therapeutic tool for treatment of ALF. Further in vivo and in vitro studies are required to optimize doze, timing, and duration of UC-MSCs and to delineate the precise molecular mechanisms underlying the protective effects UC-MSCs on ALF.

\section{Acknowledgments}

Funding: This work was supported by grants from Wuhan Municipal Science and Technology Bureau (No. 2020020601012210 and No. 2019030703011513 ).

\section{Footnote}

Reporting Checklist: The authors have completed the ARRIVE reporting checklist. Available at https://dx.doi. org/10.21037/atm-21-2885

Data Sharing Statement: Available at https://dx.doi. org/10.21037/atm-21-2885

Peer Review File: Available at https://dx.doi.org/10.21037/ atm-21-2885

Conflicts of Interest: All authors have completed the ICMJE uniform disclosure form (available at https://dx.doi. org/10.21037/atm-21-2885). ML servers as an employee of the company "Wuhan Hamilton Biotechnology Co., Ltd" and also serves as a research associate of Wuhan University. SZ serves as a full-time employee of the company "Wuhan Hamilton Biotechnology Co., Ltd". CL reports funding from Wuhan Municipal Science and Technology Bureau (No. 2020020601012210), payments were made to Wuhan University. DW serves as the managing director of "Wuhan Hamilton Biotechnology Co., Ltd" and "Guangzhou
Hamilton Biotechnology Co., Ltd"; and reports funding from Wuhan Municipal Science and Technology Bureau (No. 2019030703011513), payments were made to "Wuhan Hamilton Biotechnology Co., Ltd". The other authors have no conflicts of interest to declare.

Ethical Statement: The authors are responsible for all aspects of the work in ensuring that questions related to the accuracy or integrity of any part of the work are appropriately investigated and resolved. The study was conducted in accordance with the Declaration of Helsinki (as revised in 2013). The use of the human umbilical cord tissue from a healthy donor who gave birth and signed informed consent in Renmin Hospital was supported by the Institutional Ethics Review Board of Renmin Hospital of Wuhan University (Permit Number: WDRY2019-G001). All animal experiments were performed in accordance with the guidelines for animals care and approved by the Committee of Animals Care and Use of Hubei Provincial Center for Food and Drug Safety Evaluation and Animal Experiment (No. 2020020).

Open Access Statement: This is an Open Access article distributed in accordance with the Creative Commons Attribution-NonCommercial-NoDerivs 4.0 International License (CC BY-NC-ND 4.0), which permits the noncommercial replication and distribution of the article with the strict proviso that no changes or edits are made and the original work is properly cited (including links to both the formal publication through the relevant DOI and the license). See: https://creativecommons.org/licenses/by-nc-nd/4.0/.

\section{References}

1. Patel P, Okoronkwo N, Pyrsopoulos NT. Future Approaches and Therapeutic Modalities for Acute Liver Failure. Clin Liver Dis 2018;22:419-27.

2. Bernal W, Lee WM, Wendon J, et al. Acute liver failure: A curable disease by 2024? J Hepatol 2015;62:S112-20.

3. Volarevic V, Nurkovic J, Arsenijevic N, et al. Concise review: Therapeutic potential of mesenchymal stem cells for the treatment of acute liver failure and cirrhosis. Stem Cells 2014;32:2818-23.

4. Baroja-Mazo A, Martín-Sánchez F, Gomez AI, et al. The NLRP3 inflammasome is released as a particulate danger signal that amplifies the inflammatory response. Nat Immunol 2014;15:738-48.

5. Woolbright BL, Jaeschke H. Role of the inflammasome in 


\section{Page 12 of 13}

acetaminophen-induced liver injury and acute liver failure. J Hepatol 2017;66:836-48.

6. Wang D, Zheng J, Hu Q, et al. Magnesium protects against sepsis by blocking gasdermin $\mathrm{D} \mathrm{N}$-terminalinduced pyroptosis. Cell Death Differ 2020;27:466-81.

7. Xu B, Jiang $\mathrm{M}$, Chu $\mathrm{Y}$, et al. Gasdermin D plays a key role as a pyroptosis executor of non-alcoholic steatohepatitis in humans and mice. J Hepatol 2018;68:773-82.

8. Li Y, Xia W, Wu M, et al. Activation of GSDMD contributes to acute kidney injury induced by cisplatin. Am J Physiol Renal Physiol 2020;318:F96-F106.

9. Lee J, Choi J, Kang S, et al. Hepatogenic Potential and Liver Regeneration Effect of Human Liver-derived Mesenchymal-Like Stem Cells. Cells 2020;9:1521.

10. Huang B, Cheng X, Wang H, et al. Mesenchymal stem cells and their secreted molecules predominantly ameliorate fulminant hepatic failure and chronic liver fibrosis in mice respectively. J Transl Med 2016;14:45.

11. Chen L, Zhang J, Yang L, et al. The Effects of Conditioned Medium Derived from Mesenchymal Stem Cells Cocultured with Hepatocytes on Damaged Hepatocytes and Acute Liver Failure in Rats. Stem Cells Int 2018;2018:9156560.

12. Keating A. Mesenchymal stromal cells: new directions. Cell Stem Cell 2012;10:709-16.

13. Bongso A, Fong CY. The therapeutic potential, challenges and future clinical directions of stem cells from the Wharton's jelly of the human umbilical cord. Stem Cell Rev Rep 2013;9:226-40.

14. Lee CW, Chen YF, Wu HH, et al. Historical Perspectives and Advances in Mesenchymal Stem Cell Research for the Treatment of Liver Diseases. Gastroenterology 2018;154:46-56.

15. Zhao L, Chen S, Shi X, et al. A pooled analysis of mesenchymal stem cell-based therapy for liver disease. Stem Cell Res Ther 2018;9:72.

16. Chen G, Jin Y, Shi X, et al. Adipose-derived stem cellbased treatment for acute liver failure. Stem Cell Res Ther 2015;6:40.

17. Wang Y, Lian F, Li J, et al. Adipose derived mesenchymal stem cells transplantation via portal vein improves microcirculation and ameliorates liver fibrosis induced by CCl4 in rats. J Transl Med 2012;10:133.

18. Zhang $Z$, Lin $H$, Shi M, et al. Human umbilical cord mesenchymal stem cells improve liver function and ascites in decompensated liver cirrhosis patients. J Gastroenterol Hepatol 2012;27 Suppl 2:112-20.

19. Xiang E, Han B, Zhang Q, et al. Human umbilical
Liu et al. Mesenchymal stem cells ameliorate acute liver failure

cord-derived mesenchymal stem cells prevent the progression of early diabetic nephropathy through inhibiting inflammation and fibrosis. Stem Cell Res Ther 2020;11:336.

20. Sang JF, Shi XL, Han B, et al. Intraportal mesenchymal stem cell transplantation prevents acute liver failure through promoting cell proliferation and inhibiting apoptosis. Hepatobiliary Pancreat Dis Int 2016;15:602-11.

21. Hua D, Ju Z, Gan X, et al. Human amniotic mesenchymal stromal cells alleviate acute liver injury by inhibiting the pro-inflammatory response of liver resident macrophage through autophagy. Ann Transl Med 2019;7:392.

22. Li C, Jin Y, Wei S, et al. Hippo Signaling Controls NLR Family Pyrin Domain Containing 3 Activation and Governs Immunoregulation of Mesenchymal Stem Cells in Mouse Liver Injury. Hepatology 2019;70:1714-31.

23. Kaufmann T, Jost PJ, Pellegrini M, et al. Fatal hepatitis mediated by tumor necrosis factor TNFalpha requires caspase- 8 and involves the $\mathrm{BH} 3$-only proteins Bid and Bim. Immunity 2009;30:56-66.

24. Xu L, Zheng X, Wang Y, et al. Berberine protects acute liver failure in mice through inhibiting inflammation and mitochondria-dependent apoptosis. Eur J Pharmacol 2018;819:161-8.

25. Gehrke N, Hövelmeyer N, Waisman A, et al. Hepatocytespecific deletion of IL1-RI attenuates liver injury by blocking IL-1 driven autoinflammation. J Hepatol 2018;68:986-95.

26. Schwabe RF, Luedde T. Apoptosis and necroptosis in the liver: a matter of life and death. Nat Rev Gastroenterol Hepatol 2018;15:738-52.

27. Rolando N, Wade J, Davalos M, et al. The systemic inflammatory response syndrome in acute liver failure. Hepatology 2000;32:734-9.

28. Wen Z, Lei Z, Yao L, et al. Circulating histones are major mediators of systemic inflammation and cellular injury in patients with acute liver failure. Cell Death Dis 2016; 7:e2391.

29. Jiang $X, \mathrm{Li} Z$, Jiang $\mathrm{S}$, et al. Lipoxin $\mathrm{A} 4$ exerts protective effects against experimental acute liver failure by inhibiting the NF- $\kappa B$ pathway. Int J Mol Med 2016;37:773-80.

30. Triantafyllou E, Woollard KJ, McPhail MJW, et al. The Role of Monocytes and Macrophages in Acute and Acuteon-Chronic Liver Failure. Front Immunol 2018;9:2948.

31. Zagoura DS, Roubelakis MG, Bitsika V, et al. Therapeutic potential of a distinct population of human amniotic fluid mesenchymal stem cells and their secreted molecules in mice with acute hepatic failure. Gut 2012;61:894-906. 
32. Hu C, Zhao L, Zhang L, et al. Mesenchymal stem cellbased cell-free strategies: safe and effective treatments for liver injury. Stem Cell Res Ther 2020;11:377.

33. Negash AA, Gale M Jr. Hepatitis regulation by the inflammasome signaling pathway. Immunol Rev 2015;265:143-55.

Cite this article as: Liu M, He J, Zheng S, Zhang K, Ouyang Y, Zhang Y, Li C, Wu D. Human umbilical cord mesenchymal stem cells ameliorate acute liver failure by inhibiting apoptosis, inflammation and pyroptosis. Ann Transl Med 2021;9(21):1615. doi: 10.21037/atm-21-2885
34. Gaul S, Leszczynska A, Alegre F, et al. Hepatocyte pyroptosis and release of inflammasome particles induce stellate cell activation and liver fibrosis. J Hepatol 2021;74:156-67. 
A

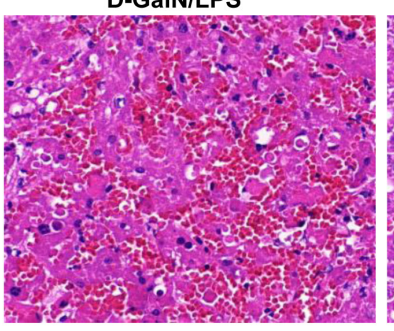

C

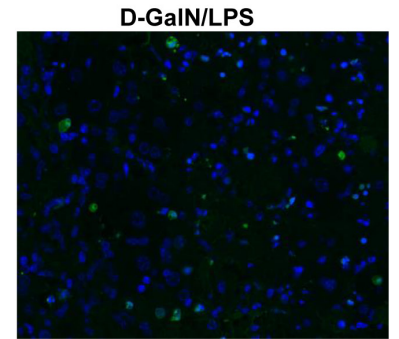

D

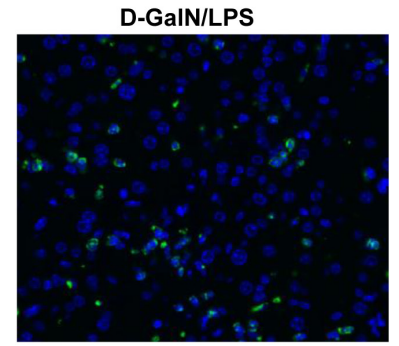

D-GaIN/LPS+UC-MSCs

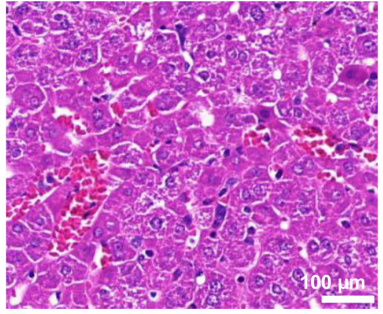

D-GaIN/LPS+UC-MSCs

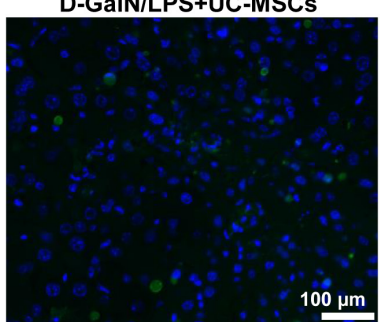

D-GaIN/LPS+UC-MSCs

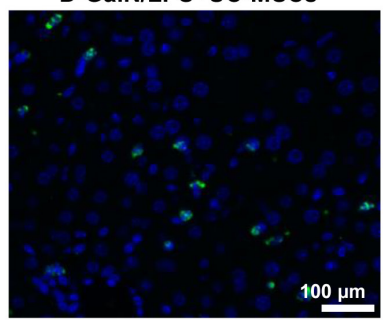

B
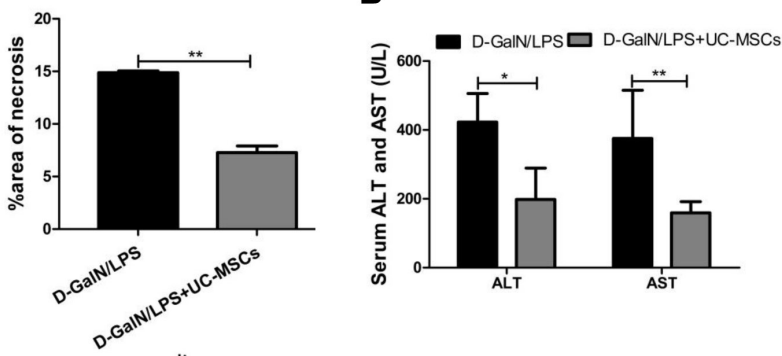
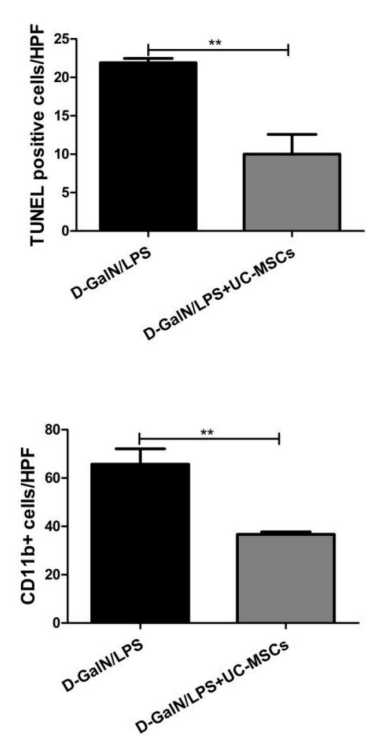

Figure S1 UC-MSCs attenuate liver injury in D-GalN/LPS-induced ALF mice. Mice were treated with D-GalN/LPS (200 mg/kg and $10 \mu \mathrm{g} / \mathrm{kg}$, respectively) and liver injury was assessed after $10 \mathrm{~h}$. UC-MSCs $\left(1 \times 10^{6}\right)$ were administered intravenously $6 \mathrm{~h}$ after D-GalN/ LPS injection ( $\mathrm{n}=6$ mice/group). (A) Hematoxylin-eosin staining of liver tissues from the D-GalN/LPS group and D-GalN/LPS+UCMSCs group. Scale bar: $100 \mu \mathrm{m}$. (B) The levels of serum ALT and AST. (C) Hepatocyte apoptosis was detected by TUNEL staining. The apoptotic cells were stained with green fluorescence and counted in randomly 5 chosen histological fields per slide (n=6 mice/group). Scale bar: $100 \mu \mathrm{m}$. (D) Representative immunofluorescence staining of the macrophage marker CD11 $\mathrm{b}^{+}$in liver sections. Quantification of $\mathrm{CD}_{11} \mathrm{~b}^{+}$per high power field. FITC-conjugated (green) secondary antibody was used. DAPI was used to visualize nuclei (blue). Scale bar: $100 \mu \mathrm{m} .{ }^{*}, \mathrm{P}<0.05 ;{ }^{* *}, \mathrm{P}<0.01$. D-GalN, D-galactosamine; LPS, lipopolysaccharide; ALF, acute liver injury; UC-MSCs, human umbilical cord mesenchymal stem cells; ALT, alanine amino transferase; AST, aspartate amino transferase; FITC, fluorescein isothiocyanate; DAPI, 4',6-diamidino-2-phenylindole. 